\title{
Appunti su semiotica, architettura e Biennale
}

di Ruggero Ragonese

Università di Modena e Reggio Emilia, Dipartimento di Comunicazione ed Economia ruggero.ragonese@unimore.it

Politecnico di Milano, Scuola del Design

ruggero.ragonese@polimi.it

\author{
Abstract \\ Some reflections on last Biennale di Architettura, starting from Elena Pirazzoli \& \\ Roberto Zancan's work, Urbs Oblivionalis. Urban Spaces and Terrorism in Italy. \\ http://www.ocula.it/files/OCULA-BIENNALE-Pirazzoli-Zancan_[12,297.304Mb].pdf
}

\section{Keywords}

Space, Architecture, Memory, Biennale 
Può essere una biennale più o meno semiotica? E prima ancora: Si può sinceramente fare questa domanda? A pensarci bene è come chiedersi se il tal Festival del cinema sia più o meno filosofico o se una Fiera d'arte sia abbastanza sociologica. Non c'è, ovviamente, un grado minore o maggiore di semioticità in un evento culturale o artistico. C'è altrettanto ovviamente un grado di semioticità rilevabile nelle intenzioni del curatore, un indirizzo generale. In questo senso la scorsa Biennale di architettura, la XIVa a Venezia, con un curatore di eccezione, Rem Koolhaas, sembrava tracciare in sé una linea ben chiara e tutta a favore di una rivisitazione interna del rapporto fra segno e spazio. Rem Koolhaas aveva scelto di porre al centro i Fundamentals, le entità minime costitutive del linguaggio architettonico e ancor più dell'abitare. Ecco allora che il padiglione centrale veniva trasformato in una specie di galleria tipologica, dove ogni ambiente rappresentava e spiegava uno dei 15 elementi costitutivi individuati. Solo che questi elementi non erano figure minimali nello stile della semiotica dei codici anni Sessanta, ma luoghi o parti dell'abitazione: pavimento, muro, soffitto, tetto, porta, finestra, facciata, balcone, corridoio, camino, bagno, scale, ascensore, rampa, scala mobile (the window, the façade, the balcony, the corridor, the fireplace, the toilet, the stair, the escalator, the elevator, the ramp.) Anche solo elencando la lista degli elementi fondamentali c'è ovviamente da interrogarsi sul criterio scelto per dettare un simile alfabeto, o dizionario, minimo. Ci si chiede come a livello categoriale la finestra e la porta possano stare insieme alla facciata di cui, a tutta prima, dovrebbero essere parti costitutive o come possano essere presenti nell'elenco degli elementi quali la scala mobile e il camino e non per esempio la colonna che come i precedenti non rappresenta uno spazio, ma un elemento, appunto, di questo. Poco importa, la risposta la si trova agevolmente nei semplici e spartani libricini che racchiudono la ricerca coordinata da Koolhaas e altri architetti e condotta dagli studenti dell'Harvard graduate School of Design come la si trovava, specularmente, nelle stanze del Padiglione centrale. Appare chiaro così che dietro gli Elements of Architecture si nasconde tutt'altro che una segmentazione dei tratti di tipo linguistico, quanto invece una carrellata storica di diverse tipologie di spazi o parti architettoniche. La ricerca dei fondamentali si risolve quindi in diversi significati storicizzati e nei canoni teorici che si sono via via succeduti (da Alberti a Kahn a Rossi). Che l'architettura si rappresenti come svincolata dall'antica illusione verbocentrica potrebbe essere un bene, se non che qui non è successo questo, ma piuttosto un travisamento di quel procedere: segni e figure restano ma solo come comparse per un discorso che invece prende tutt'altre pieghe. Tutto perfettamente in linea con una certa mistificazione concettuale che risolve il segno nel suo uso storico, in una ricerca costante di categorie abbastanza larghe estensionalmente da poter coprire i limiti intensionali. Se la XIVa biennale doveva essere una "riflessione sull'architettura e non sugli architetti" come ha detto Koolhaas alla presentazione dell'evento, questa riflessione ha mostrato una certa fossilizzazione di massima e un incredibile attaccamento al concetto di segno. La preoccupazione strutturale di 


\section{Ocula ${ }^{\text {Flux__aggi }}$}

Ruggero Ragonese | Appunti su Semiotica, Architettura e Biennale

definire le unità minime del sistema data come un tutto omogeneo permane, ma al contempo queste articolazioni si presentano prive degli ancoraggi logici che, almeno, le suddivisioni, spesso arzigogolate, degli studi di semiotica dell'architettura anni Sessanta garantivano. Data la stura a un velo di 'scientificità' tutto è quindi introducibile dentro macro categorie sedicenti fondamentali. Niente di strano e neanche niente di male, anche se il risultato complessivo oltre una naturale immediata fascinazione (il padiglione centrale metteva a confronto in modo assai interessante modelli, riproduzione, plastici, opere vere e proprie) rischia di restare incomprensibile nella sua parte più teorica e di ricerca. A questo punto appare chiaro che difficilmente si può pretendere un passaggio dal 'sistema architettura', ristretto in una dimensione nebulosa, alle singole strutturazioni operate da eventuali codici. Così paradossalmente il rapporto prossemico e spaziale fra soggetto e architettura resta relegato tutto alla rievocazione storica catturata in diverse e svincolate istantanee e rappresentazioni (abbondano miniature, stampe, disegni). Così segno è ancora una volta per Koolhaas quello che chi lo usa intende per segno.

Disiecta membra, i fundamentals sono sembrati così piuttosto una raccolta retorica di strategie efficaci o di fortune normative (quale altezza, quale lunghezza, quale peso), un prontuario giustamente progettuale che però non rende conto della ricchezza semiotica complessiva di un'opera o addirittura la riduce a una parte o a una sua memoria. La parte chiamata Monditalia alle Corderie, di cui di seguito presentiamo un interessante articolo di due dei diversi curatori chiamati a gestire i singoli spazi, di fatto completava in chiave nazionale questa prospettiva. 41 installazioni alle Corderie dell'Arsenale unite da una enorme riproduzione della Tabula Peutingeriana che scandiva le aree espositive divise per aria geografica. Il curatore della mostra Ippolito Pestellini Laparelli, del gruppo Amo, aveva sottolineato l'importanza e la "specifica volontà di mettere in relazione l'architettura con altre discipline". Questo Tour d'Italie aveva, al di là dei suoi singoli episodi, un suo interesse perché incentrato sulla rifunzionalizzazione e la risemantizzazione di spazi disseminati nella Penisola italiana e anche al di fuori di essa (i resti del colonialismo italiano in Libia o in Eritrea). Anche qui il cuore del segno architettonico appariva in un primo tempo estromesso, ma riusciva a trapelare in un repertorio di luoghi che avevano perso, per dirla con Barthes, le loro denotazioni primarie a favore di connotazioni differenti provocate da usi e progettualità molteplici (case diventate templi induisti, fabbriche abbandonate, discoteche riutilizzate). Uno shift semantico continuo che sembrava spostarsi un poco a ogni passo fatto sulla mappa Peutingeriana. Il testo di Pirazzoli e Zancan che hanno presentato il loro intervento in uno di questi all'interno di Monditalia (il titolo: Urbs oblivionalis. Urban Spaces and Terrorism in Italy) ha, in questo senso, il merito di muoversi in un senso originale, invertendo i poli della discussione e del problema della memoria. Di fronte al minuzioso elenco e alle varie immagini che testimoniano dei luoghi del Terrorismo in Italia, Pirazzoli e Zancan scelgono di fermarsi e chiedersi non cosa 


\section{Ocula \\ Ruggero Ragonese | Appunti su Semiotica, Architettura e Biennale}

resti della memoria nell'architettura (tema usurato), ma cosa resti dell'architettura dopo la memoria. Questo poi è il motivo per cui agli albori di una nuova biennale e dopo due anni proponiamo su Ocula questo testo. E' una sintesi originale della Biennale scorsa e un buon viatico per una riflessione sulla prossima che inizierà a breve. In una sequela di riferimenti fintamente semiotici, di rimandi pseudo linguistici, Pirazzoli e Zancan ci offrono, da non semiotici, qualche spunto di riflessione interessante. Non a caso lo fanno con un riferimento a Eco che suona come una sorta di sutura fra un discorso semiotico complesso e i vasti studi culturali sulla memoria che hanno sempre più preso piede negli ultimi anni. L'Ars oblivionalis proposta da Eco in un articolo su Repubblica del 2006 e che i curatori riprendono a proposito dei luoghi delle stragi terroristiche in Italia, attentamente riletti nel loro spazio espositivo, colma quindi la distanza fra il segno troppo sfuggente e arbitrario dei Fundamentals e la ricerca fossilizzata di 'reali' elementi discreti capaci di formare articolazioni del linguaggio. L'Ars oblivionalis infatti "dovrebbe porre i principi non per ovviare a, ma per produrre il deterioramento della traccia attraverso un progetto volontario". Interrogarsi su come eleminare una memoria e su come questo sia possibile, è una base per riprodurre segni e per evitare i cataloghi. Ovviamente, deteriorare la traccia non significa cancellarla, come dimenticare non significa perdere un ricordo: significa invece introiettarla in una rete di segni fino a renderla quasi irriconoscibile. "Dimenticare per fare affiorare il ricordo. Ciò è tanto più vero per la 'stagione del terrorismo', dove luoghi, edifici e muri diventavano obiettivi proprio in virtù delle loro qualità di luoghi comuni, quotidiani, banali.” La traccia insomma non si cancella, ma non può nemmeno assurgere al centro della riflessione sull'architettura, se no, semplicemente, non sarebbe una traccia (o non sarebbe architettura). Essa fa parte integrante di una organizzazione testuale che modula e articola anche gli elementi più propriamente tipologici della struttura architettonica (i fundamentals per usare il termine di Koolhaas). L'analisi come il progetto non può che produrre strumenti che il più possibile cerchino in modo rigoroso di considerare a livello sistemico tutte gli elementi in gioco nel testo spaziale. Questo inevitabilmente impone di uscire finalmente dalla logica del singolo significante e considerare invece insiemi più vasti che includano spazi, soggetti e oggetti. Speriamo di iniziare questo non semplice lavoro anche con gli spunti e il materiale che troveremo alla XVa Biennale. 